\title{
Polarization Fluctuations Demonstrate Nonlinear Anisotropy of a Vertical-Cavity Semiconductor Laser
}

\author{
M.P. van Exter, A. Al-Remawi, and J.P. Woerdman \\ Huygens Laboratory, Leiden University, P.O. Box 9504, 2300 RA Leiden, The Netherlands
}

(Received 12 September 1997)

\begin{abstract}
We report observation of polarization fluctuations in vertical-cavity semiconductor lasers, which allows us to demonstrate and quantify the importance of nonlinear polarization anisotropy. We focus on three aspects, which all fit within the same theoretical framework: (i) a nonlinear spectral redshift, (ii) an extra four-wave mixing peak in the optical spectrum, and (iii) correlations between the polarization fluctuations. [S0031-9007(98)06185-7]

PACS numbers: $42.55 . P x$
\end{abstract}

In conventional semiconductor lasers the optical polarization is pinned by the stripe geometry. In semiconductor vertical-cavity surface-emitting lasers (VCSELs) such pinning is practically absent due to their nominal cylindrical symmetry. As a consequence it has been predicted that the polarization fluctuations in the emitted light, being driven by spontaneous emission noise and modified by the optical anisotropies, can become exceptionally strong in these lasers $[1,2]$. Earlier studies showed the dominant anisotropy in VCSELs to be linear birefringence caused by stress acting via the elasto-optic effect [3] and by internal electric fields acting via the electro-optic effect [4]. Nonlinear anisotropies, which increase with laser power and reflect the polarization dependence of the gain saturation, were found to be much weaker and noticeable only as small deviations from a linear coupled-mode description [5]. Nonlinear anisotropies have been mentioned as the prime origin of polarization switches, i.e., sudden changes in the VCSEL polarization as a function of laser current [6], but quantitative data is scarce [7] and competing mechanisms might also play a role [8,9]. In this Letter we will show how fluctuations in the laser polarization presents conclusive experimental evidence, qualitative and quantitative, for the existence of nonlinear anisotropies (or polarization-dependent saturation) in VCSELs.

The simplest and still realistic description of the nonlinear aspects of a quantum-well VCSEL is based on a model developed by San Miguel et al. [10], and extended by others $[1,2,6,11]$, in which the conduction and heavy-hole valence band are treated as four discrete levels, $M= \pm \frac{1}{2}$ and $M= \pm \frac{3}{2}$, interacting via the circular components of the optical field. An important parameter in this model is $\Gamma$, which describes the spin flip relaxation between levels with opposite angular momentum, $+M$ and $-M\left(\Gamma=\gamma_{s} / \gamma\right.$, where $\gamma_{s}$ and $\gamma$ are the decay rates of the spin-difference and spin-averaged inversions, respectively). The parameter $\Gamma$ determines the strength of the nonlinear anisotropies; for small $\Gamma$ the circularly polarized optical transitions are almost decoupled and the optical saturation is highly anisotropic; for $\Gamma \rightarrow \infty$ the system effectively reduces from four to two levels and the nonlinear anisotropy disappears. For $\Gamma \gg 1$, a case that applies to our experiments (see below), the spin-difference inversion can be adiabatically eliminated from the laser rate equations [1,2] and the polarization dynamics simplifies to that of a class A laser [12]. For such a laser one can show that, irrespective of the microscopic model, there is only one (complex-valued) parameter that determines the nonlinear anisotropies [2].

A complete description of the light emitted by a singletransverse-mode VCSEL involves four variables, which can be chosen as phase, intensity, polarization direction, and polarization ellipticity. When we neglect the phase and assume the intensity to be constant (operation sufficiently far above threshold), we retain the polarization angle $\phi(t)$ and the ellipticity $\chi(t)$. For practical VCSELs the optical field is approximately linearly polarized [5], along the axis of linear birefringence which we define to be the $x$ axis, so that the optical field can be written as $\vec{E}(t) e^{-i \omega_{l} t} \approx|\vec{E}|\left[\vec{e}_{x}-(\phi+i \chi) \vec{e}_{y}\right] e^{-i \omega_{l} t}$, with $\phi, \chi \ll 1$. For $\Gamma \gg 1$ and constant $|\vec{E}|$ the basic rate equations for the polarization angles are $[1,2]$

$$
\begin{gathered}
\frac{d \phi}{d t}=2 \epsilon \phi-\left(2 \sigma+2 \alpha \frac{\kappa \mu}{\Gamma}\right) \chi+f_{\phi}, \\
\frac{d \chi}{d t}=2 \sigma \phi+\left(2 \epsilon-2 \frac{\kappa \mu}{\Gamma}\right) \chi+f_{\chi} .
\end{gathered}
$$

The linear anisotropies appear as a linear birefringence $2 \sigma$ and a linear dichroism $2 \epsilon$, where $\sigma>0$ and $\epsilon>$ 0 correspond to $x$-polarized light having the highest frequency and highest loss, respectively. The nonlinear anisotropies appear as $\kappa \mu / \Gamma$, in Eq. (1a) multiplied by the phase-amplitude coupling parameter $\alpha$, where $\kappa$ is the cavity loss rate of the optical field and $\mu$ is the pump parameter $\left[\mu \approx\left(I-I_{\mathrm{th}}\right) / I_{\mathrm{th}}\right]$, which measures the degree of saturation. The Langevin noise sources $f_{\phi}$ and $f_{\chi}$ represent the spontaneous emission that perturbs the system away from the $x$-polarized equilibrium state $(\phi=\chi=0)$ and drives the polarization fluctuations $\phi(t), \chi(t) \ll 1$. 
The experiments were performed on a batch of some 50 proton-implanted GaAs quantum-well VCSELs operating at a wavelength of $850 \mathrm{~nm}$ [13]. To facilitate comparison of the various experimental approaches (see below) we will display results obtained for one VCSEL only. The linear birefringence of the chosen VCSEL was relatively small $(\approx 3 \mathrm{GHz})$, to ensure that the nonlinear anisotropy was not completely overwhelmed by the linear anisotropy. Figure 1 shows the polarization-resolved output power of this VCSEL as function of input current. Single transverse-mode operation was obtained between threshold $\left(I_{\mathrm{th}}=4.5 \mathrm{~mA}\right)$ and $I=10.5 \mathrm{~mA}$. Near $I \approx 9 \mathrm{~mA}$ this VCSEL exhibits a polarization switch with hysteresis.

We will give three experimental proofs for the existence of nonlinear anisotropies in VCSELs. The first proof comes from the optical spectrum, where polarization fluctuations show up as a weak $y$-polarized peak, which contains $\approx 1 \%$ of the intensity of the dominant $x$-polarized peak [see Fig. 1(a)]. The $y$-polarized peak is shifted and broadened with respect to the $x$-polarized peak [5], the shift and broadening being determined by the imaginary and real parts of two eigenvalues of Eqs. (1a) and (1b), which are $\lambda_{1,2}=2 \epsilon-(\kappa \mu / \Gamma) \pm i \omega_{0}$, where $\omega_{0}^{2}=4 \sigma^{2}+4 \sigma \alpha \kappa \mu / \Gamma-(\kappa \mu / \Gamma)^{2}$. These eigenvalues show how nonlinear anisotropies, as quantified by

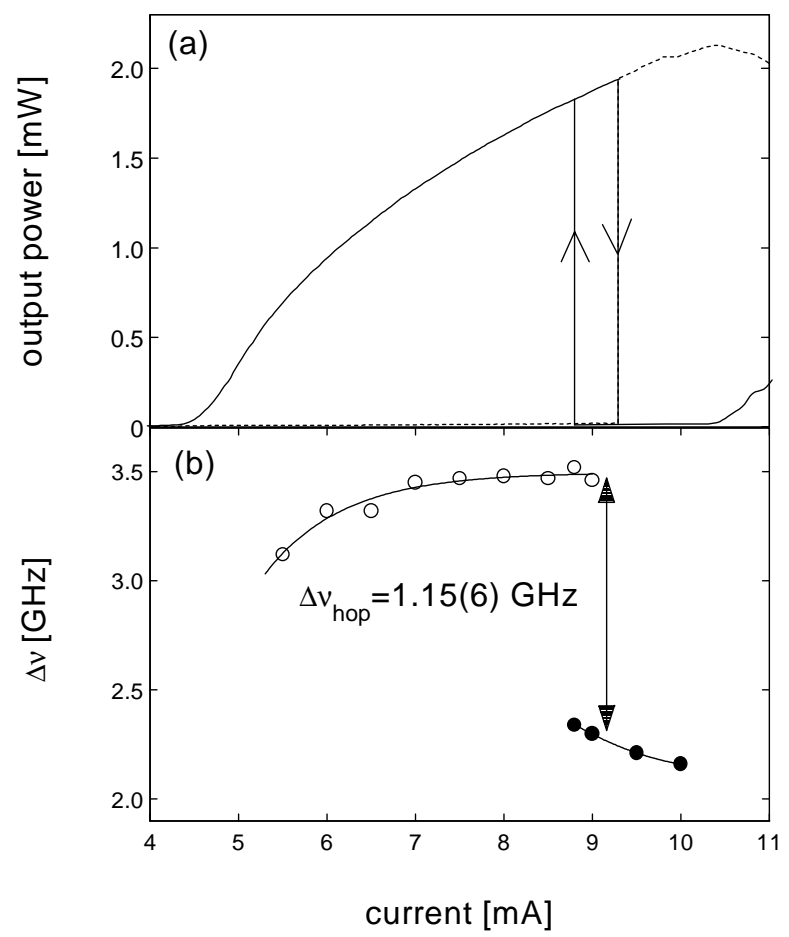

FIG. 1. Polarization-resolved output power (a) and effective birefringence $\Delta \nu$ (b) as a function of current. At low current the emission is dominantly $x$ polarized (solid curve, open circles); at higher currents the laser switches to $y$-polarized emission (dashed curve, dots); above $10.5 \mathrm{~mA}$ higher-order transverse modes appear. Note the sudden change in $\Delta \nu$ associated with the polarization switch. $\kappa \mu / \Gamma$, are expected to lead to an additional damping and redshift of the "nonlasing" component $[2,14]$. The nonlinear redshift shows up in its purest form around a polarization switch, where the nonlinear contribution changes sign but the linear one does not, so that the frequency splitting $\Delta \nu$ between the two spectral peaks is expected to change by an amount [15]

$$
\Delta \omega_{\text {hop }} \approx 2 \alpha \frac{\kappa \mu}{\Gamma} .
$$

Figure 1(b) shows the frequency splitting $\Delta \nu$ (measured with a planar Fabry-Perot interferometer) as a function of current. When the laser polarization switches, $\Delta \nu$ was found to jump from $3.45 \mathrm{GHz}$ for dominant $x$-polarized emission to $2.30 \mathrm{GHz}$ for dominant $y$-polarized emission. We attribute this jump to the nonlinear redshift mentioned earlier; it has been observed for all our VCSELs that exhibit a polarization switch [9]. In all cases the sign of the observed frequency jump agreed with that predicted for a nonlinear redshift and confronting its magnitude with Eq. (2) we obtain $\alpha(\kappa \mu / \Gamma) \approx \Delta \omega_{\text {hop }} / 2=3.6(2) \mathrm{ns}^{-1}$.

The second experimental proof also comes from the optical spectrum. We write down the deterministic (i.e., noise-free) time evolution of the optical polarization as

$$
\begin{aligned}
& \phi(t)+i \chi(t)=e^{[2 \epsilon-(\kappa \mu / \Gamma)] t}\left(e^{i \omega_{0} t}+A e^{-i \omega_{0} t}\right), \\
&|A|^{2} \approx\left(\alpha^{2}+1\right)\left(\frac{\kappa \mu}{2 \omega_{0} \Gamma}\right)^{2} \ll 1,
\end{aligned}
$$

where we again assumed the nonlinear effects to be relatively weak. The evolution of $(\phi+i \chi)$ consists of a "corotating" term and a (generally much weaker) "counterrotating" term $[1,2]$. In the optical spectrum the corotating term is visible as the $y$-polarized peak that we just discussed, which is displaced by $-\omega_{0}$ with respect to the dominant $x$-polarized laser mode. The counterrotating term corresponds to a spectral peak displaced by $+\omega_{0}$, i.e., a mirror image of the "nonlasing mode" with respect to the lasing mode. The presence of this mirror image is a direct result of nonlinear anisotropies; it can be seen as a polarization-type of four-wave mixing (FWM) in the laser, where the polarization beat between the lasing and nonlasing modes drives an oscillating spin population, which then scatters part of the $x$-polarized lasing mode into two $y$-polarized components, one displaced by $-\omega_{0}$, i.e., into the nonlasing mode, and the other by $+\omega_{0}$, which creates the FWM component. In view of this theoretical prediction of the FWM component we have made a detailed search of the spectra of our VCSELs, especially those with small $\omega_{0}$ [see Eq. (3b)].

Figure 2 shows the optical spectrum of the $y$-polarized emission for $I=9.0 \mathrm{~mA}$ (and $1.9 \mathrm{~mW}$ of optical output). For this measurement the $x$-polarized lasing mode (peak $x$ ), which by itself peaks at 40 on this scale, was suppressed to about $10^{-5}$ for the dashed curve (to 


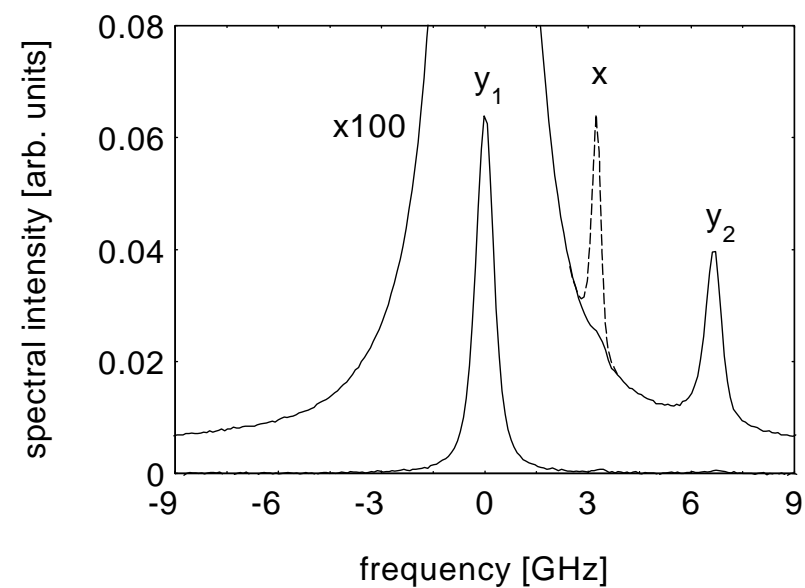

FIG. 2. A detailed view of the $y$-polarized optical spectrum at $I=9.0 \mathrm{~mA}$. For the solid curve the $x$-polarized lasing mode was fully suppressed; for the dashed curve suppression by a factor of $10^{5}$ allows it to serve as a marker. Note the presence of the "nonlasing peak" $y_{1}$ and the FWM peak $y_{2}$.

serve as a frequency marker) and to less than $10^{-6}$ for the solid curves. The $100 \times$ magnification clearly shows the presence of a weak FWM peak (peak $y_{2}$ ), being the mirror image of the usual nonlasing peak (peak $y_{1}$ ). In Fig. 2 the relative strength of $y_{2}$ as compared to $y_{1}$ is $0.63(7) \%$. Inserting this value into Eq. (3b) and combining it with the measured frequency splitting $\omega_{0} /(2 \pi)=3.45 \mathrm{GHz}$, we find $(\kappa \mu / \Gamma) \sqrt{\alpha^{2}+1}=3.5(4) \mathrm{ns}^{-1}$. This value agrees with the earlier value obtained from the nonlinear redshift, since typically $\alpha=3-4$, so that $\sqrt{\alpha^{2}+1} \approx \alpha$.

Figure 3 shows the measured intensity of the FWM peak, relative to that of the nonlasing peak, as a function of current; the open circles denote the case of dominant $x$-polarized emission, and relatively large effective birefringence $\omega_{0}$, whereas the solid dots denote the case after the polarization switch, where $\omega_{0}$ is smaller. The

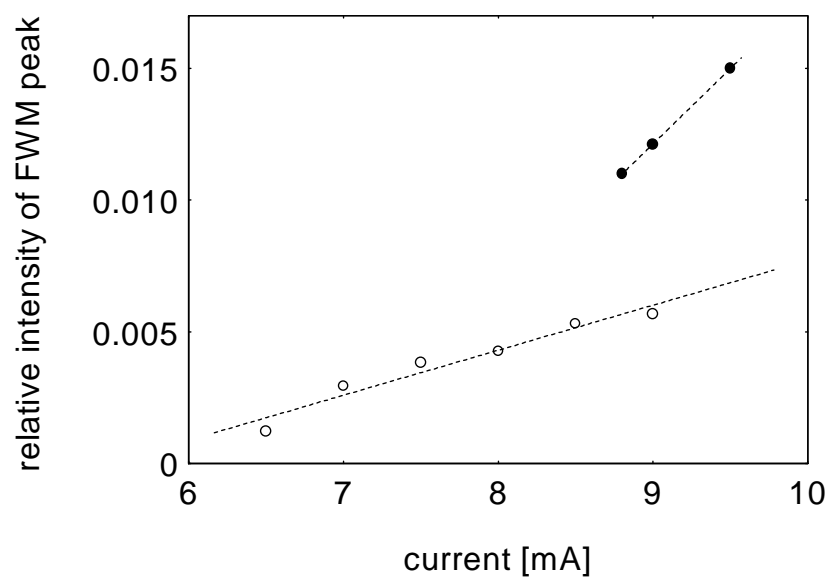

FIG. 3. The spectral intensity of the FWM peak relative to the nonlasing peak, plotted as a function of current. The circles and dots correspond to the situation before and after the polarization switch, respectively. The dashed lines are to guide the eye. relative strength of the FWM peak varies as expected from Eq. (3b): it is most prominent for small $\omega_{0}$ and increases steeply with current, i.e., with the amount of saturation [16].

FWM peaks were also observed for VCSELs with different frequency splittings $\Delta \nu=\omega_{0} /(2 \pi)$ : for a laser with $\Delta \nu$ changing from 6.7 to $5.7 \mathrm{GHz}$ at a switching current of $I=9.5 \mathrm{~mA}$, the relative intensity of the FWM peak was $y_{2} / y_{1}=0.15(3) \%$, in agreement with theory. For a laser with a much smaller splitting, i.e., $\omega_{0} /(2 \pi)=$ $-0.6 \mathrm{GHz}$, the relative intensity of the FWM peak was as much as $20 \%$. For this laser the assumption of relatively weak nonlinear anisotropies clearly breaks down, and the approximate expressions cannot be used.

The third experimental proof of nonlinear anisotropies comes from a quantitative comparison between the fluctuations in the polarization direction $\phi$ and the ellipticity $\chi$. The presence of a counterrotating term in Eq. (3a) implies that the evolution of $\phi+i \chi$ in the complex plane is not along circles, but instead along elliptical trajectories. As a result, when the system is driven by noise, the fluctuations in $\phi$ and $\chi$ are predicted to have different amplitudes [1]. From Eq. (3) one already obtains the ratio between the directional and ellipticity fluctuations for frequencies $\omega \approx \omega_{0}$. Fourier analysis of the original Eqs. (1a) and (1b), in the presence of white noise, shows that this ratio actually depends on noise frequency as [15]

$$
\frac{\left\langle|\phi(\omega)|^{2}\right\rangle^{\frac{1}{2}}}{\left\langle|\chi(\omega)|^{2}\right\rangle^{\frac{1}{2}}} \approx 1+\frac{2 \alpha \kappa \mu}{\omega_{0} \Gamma}\left(\frac{\omega_{0}^{2}}{\omega_{0}^{2}+\omega^{2}}\right) .
$$

We have measured the polarization fluctuations in the VCSEL light, by passing the light through a (rotatable) $\lambda / 4$ plate and polarizer, to project onto a (selectable) polarization state, and by measuring the intensity fluctuations in that projection with a fast photodiode and RF analyzer (up to $3.7 \mathrm{GHz}$ ). The $\lambda / 4$ plate and polarizer allow projections on any polarization state so that we can observe fluctuations in $\phi$ or $\chi$ or any combination of the two. The lower curve in Fig. 4 shows the noise spectrum at $I=9.0 \mathrm{~mA}$ for projection, with a polarizer only, onto the dominant $x$ polarization, so that we observe the intensity noise in the $x$ polarization, $\left\langle\left|P_{x}(\omega)\right|^{2}\right\rangle^{\frac{1}{2}}$. The upper and middle curves show the noise spectrum for projection onto the $x+y\left(45^{\circ}\right.$ linear) and $x+i y$ (circular) polarization, respectively. As the noise level in the mixed projections is much larger than that in the $x$ projection, the extra noise must originate from polarization instead of intensity fluctuations. The coherent beat between the two polarized components yields $\phi(t)$ (upper curve) and $\chi(t)$ (middle curve) as the in- and out-of-phase component of the $y$-polarized light with respect to the dominant $x$-polarized light [see expression for $\vec{E}(t)$ above Eqs. (1a) and (1b)]. The upper two curves in Fig. 4 thus confirm the theoretical prediction [1] that the polarization fluctuations are stronger in the polarization direction $\phi$ than in 


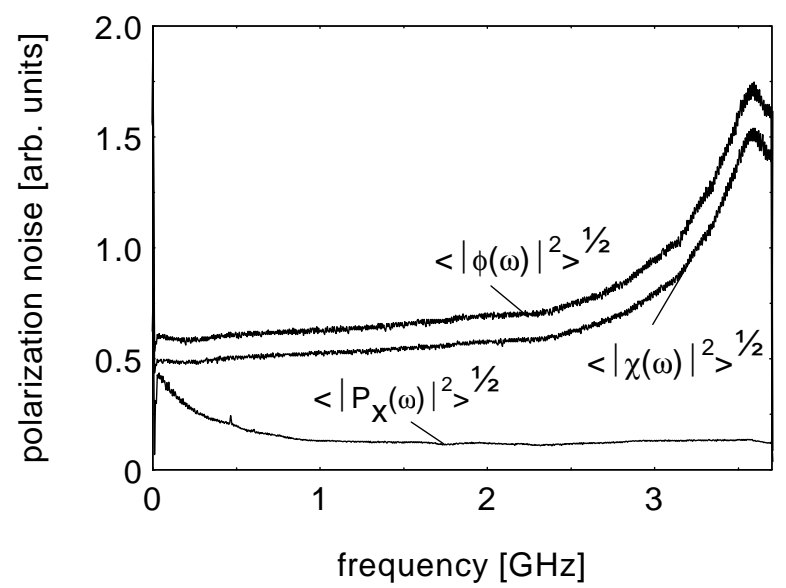

FIG. 4. The frequency-dependent intensity noise as measured behind a polarizer (lower curve) or a combination of $\lambda / 4$ plate and polarizer (upper curves).

the ellipticity $\chi$. Specifically, we find $|\phi(\omega) / \chi(\omega)|=$ $1.25(4)$ at low frequency $(0.5 \mathrm{GHz})$ and $1.11(3)$ at the resonance frequency of the polarization beat $(\Delta \nu \approx$ $3.6 \mathrm{GHz})$. This corresponds to $\alpha(\kappa \mu / \Gamma)=2.7(4) \mathrm{ns}^{-1}$, being close to the values found above. When we forced the laser to switch polarization, by increasing and then decreasing the current, we found that the fluctuations in $\chi$ became more prominent than those in $\phi$ (not shown). This is consistent with Eq. (4); the dominant polarization fluctuations change character when $\omega_{0}$ changes sign, the asymmetry being a consequency of $\alpha$. Analysis of this case leads to a similar value for $\alpha(\kappa \mu / \Gamma)$, namely, 3.2(4) $\mathrm{ns}^{-1}$.

In conclusion we have demonstrated the existence of nonlinear anisotropy in VCSELs, in three different ways, using a detailed study of the polarization fluctuations as a diagnostic tool. For proton-implanted VCSELs at typical drive currents we determined the nonlinear anisotropy $\alpha(\kappa \mu / \Gamma)$ as 3-4 $\mathrm{ns}^{-1}$, which is an order of magnitude smaller than the typical linear birefringence in these devices. An estimate of $\Gamma$ is considerably hindered by large uncertainties in $\alpha, \kappa$, and $\mu$. If we take $\alpha=3-4$, $\kappa=133-600 \mathrm{~ns}^{-1}$, and $\mu \approx 1$, we obtain as a rough estimate $\Gamma=100-800$.

We thank R.F.M. Hendriks for fruitful discussions. We acknowledge support of the "Stichting voor Fun- damenteel Onderzoek der Materie (FOM)" and of the European Union in the ESPRIT Project No. 20029 (ACQUIRE) and the TMR network ERB4061 PL951021 (Microlasers and Cavity QED).

[1] H. F. Hofmann and O. Hess, Quantum Semiclass. Opt. 10, 87 (1998).

[2] M.P. van Exter, R.F.M. Hendriks, and J.P. Woerdman, Phys. Rev. A 57, 2080 (1998).

[3] A.K. Jansen van Doorn, M.P. van Exter, and J.P. Woerdman, Appl. Phys. Lett. 69, 1041 (1996).

[4] R.F.M. Hendriks, M.P. van Exter, J.P. Woerdman, A. van Geelen, L. Weegels, K. H. Gulden, and M. Moser, Appl. Phys. Lett. 71, 2599 (1997).

[5] A. K. Jansen van Doorn, M. P. van Exter, A. M. van der Lee, and J. P. Woerdman, Phys. Rev. A 55, 1473 (1997).

[6] J. Martin-Regalado, F. Prati, M. San Miguel, and N. B. Abraham, IEEE J. Quantum Electron. 33, 765 (1997).

[7] J. Martin-Regalado, J.L.A. Chilla, J. J. Rocca, and P. Brusenbach, Appl. Phys. Lett. 70, 3350 (1997).

[8] K. D. Choquette, R. P. Schneider, Jr., K. L. Lear, and R. E. Leibenguth, IEEE J. Sel. Top. Quantum Electron. 1, 661 (1995).

[9] The switch in Fig. 1 is from high-frequency $x$ polarization to low-frequency $y$ polarization when increasing the current; however, other lasers were observed to switch from low to high frequency. This suggests that nonlinear anisotropy is not the main switching mechanism. We suspect that the current dependence of the linear dichroism plays a more important role.

[10] M. San Miguel, Q. Feng, and J. V. Moloney, Phys. Rev. A 52, 1728 (1995).

[11] M. Travagnin, M.P. van Exter, A. K. Jansen van Doorn, and J. P. Woerdman, Phys. Rev. A 54, 1647 (1996); 55, 4641(E) (1997).

[12] C. O. Weiss and R. Vilasceca, Dynamics of Lasers (VCH, Weinheim, 1991).

[13] Vixel Corporation, 325 Interlocken Parkway, Broomfield, CO 80021, model PRI-LA-S-850-1x8-3S.

[14] H. van der Lem and D. Lenstra, Opt. Lett. 22, 1698 (1997).

[15] Here we assumed that $\alpha \kappa \mu / \Gamma \ll 2 \sigma$ and $\alpha \gg 1$ (typically $\alpha=3-4$.

[16] A direct comparison with Eq. (3b) is hindered by the appreciable deviation from linearity between output power and laser current; the laser efficiency decreases with current as is generally the case for VCSELs. 